\title{
Exciton patterns in molecular dots and wires and application to polymer morphology
}

\author{
Raoul Kopelman, Zhong-You Shi and Ching Shan Li \\ Department of Chemistry, University of Michigan, Ann Arbor, MI 48109, USA
}

\begin{abstract}
Time sequencing of laser excitation can control the microscopic patterns of molecular excitations (excitons) in nanometer scale molecular ensembles. This is demonstrated for isolated guest chains in polymer blends (P1VN/PMMA) and for molecular clusters in molecularly doped polymers (naphthalene/PMMA). The subwavelength exciton patterns are monitored via time resolved luminescence and fusion kinetics. The heterogeneity exponent $(h)$ is 0.5 for isolated P1VN chains, zero (classical) for pure P1VN and "fractal-like" throughout certain concentration regimes. Correlation is made with morphology changes (phase separation, filamentation). At concentrations below $0.01 \%$, the excitons are constrained to a truly one-dimensional topology (wire). At higher concentrations there is a fractal-like topology which may be associated with spinoidal decomposition. Similar studies were conducted on naphthalene-doped PMMA (1-20 wt \%). The lower concentration samples are neither segregated nor random solution phases. They probably consist of islands (dots) of naphthalene aggregates. The exciton distribution in such islands is non-Poissonian under steady-state excitation or under pulse excitation with delay times, resulting in anomalous exciton fusion kinetics.
\end{abstract}

\section{Introduction}

It is well known that spatial photon patterns can create spatial excitation (exciton) patterns in a condensed medium - the production of transient gratings [1] being one such example. We note that such patterns are not finer than $\lambda / 2$, i.e. the optical diffraction limit for the given photon wavelength $\lambda$. On the other hand, reaction kinetics effects in restricted domains can produce patterns of the order of $\rho^{-1}$ where $\rho$ is the particle density [2]. Specifically, the exciton reaction kinetics (fusion, trapping) is driven by the dynamics of exciton creation, which in turn is determined by the temporal pattern of the exciting radiation field. For instance, an ultrashort laser pulse will essentially create an instantaneous random distribution of excitons in a small domain (smaller than $\lambda$ ). On the other hand, a steady-state excitation may result in an ordered distribution [2-4]. This enables one to transform patterns from the time domain into the spatial domain (and vice versa).

An easy method for the detection of the ultrasmall spatial patterns is based on the "reverse transformation" of spatial patterns into temporal patterns. Exciton fusion kinetics and luminescence decay provides such a tool. For instance, on a molecular wire, a random exciton distribution results in the familiar second order exciton fusion kinetics $[2,3]$. On the other hand, an ordered distribution (e.g., superlattice) of excitons will result in third order kinetics [2].

\section{Experimental}

Experimentally, we follow the phosphorescence and delayed fluorescence decays of selected samples. The two examples discussed are: (1) the isolated poly-1-vinyl-naphthalene (P1VN) molecular chains in extremely dilute P1VN/PMMA blends, and (2) the isolated clusters of naphthalene embedded in dilute naphthalene/PMMA glasses.

The systems, procedures and instrumentation have been discussed in detail [5-7]. The same is true for the computer simulations $[2,8]$.

\section{Molecular wires}

The most dilute blends $(0.005 \%$ and $0.01 \%$ weight) show a kinetic behavior of the form

$F \sim t^{-1 / 2} P$, 
where $F$ is the delayed fluorescence and $P$ the phosphorescence, as can be seen from fig. 1 . This is a case of exciton heterofusion [2,6-8], involving free excitons and trapped excitons (at excimer sites).

According to a recent theoretical analysis $[9,10]$, a behavior following eq. (1) means that there are gaps (depletion zones) around each trapped exciton with the nearest-neighbor distribution of distances $r$ (between the free and trapped excitations) given by a "skewed-Gaussian" distribution

$f(r) \sim r \mathrm{e}^{-2 \gamma r}$,

rather than a Poisson (random) distribution of the form

$f(r) \sim \mathrm{e}^{-\beta r}$.

At long times, or with steady-state excitation, most chains will have a "self-organized" distribution given by eq. (2).

The higher concentration samples show a kinetic behavior of the form

$F \sim t^{-h} P$

for longer-time decays (allowing enough time for
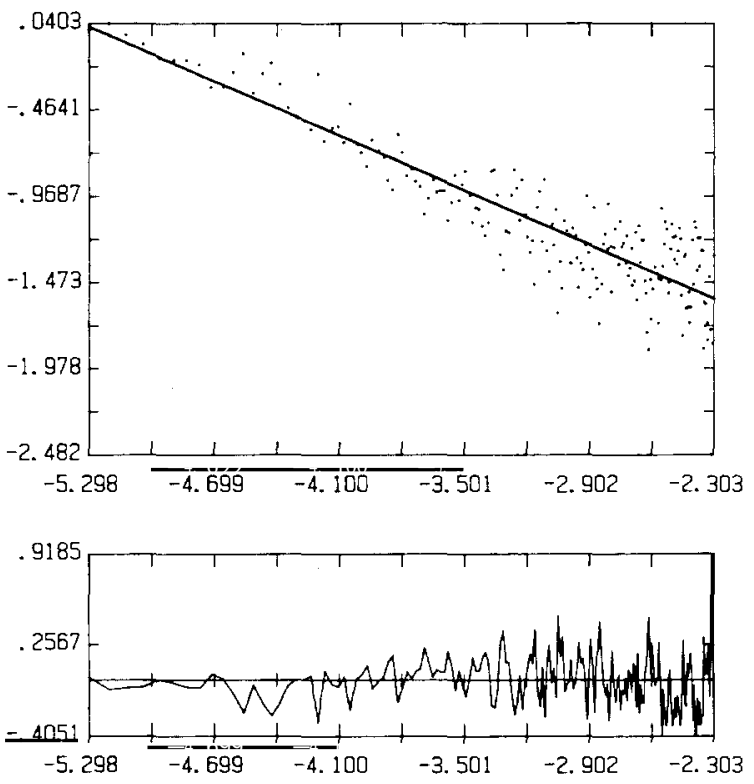

$\operatorname{Ln}[$ Time (Second) ]

Fig. 1. $\ln (F / P)$ vs. In $t$ for $0.01 \%$ blend. $h=0.53 \pm 0.03$ the formation of trapped excitons and heterofusion kinetics). However, for shorter times;

$F \sim t^{-h} P^{2}$

(this homofusion regime cannot be analyzed for the very dilute samples due to reduced signal/noise). The heterogeneity (fractal) exponent $h$ varies between $\frac{1}{2}$ and zero (classical value). The non-monotonic behavior of $h$ with concentration has been related with morphology changes and phase separations [6] and may be related to spinoidal decomposition.

\section{Molecular dots}

An experimental system representing "dots", i.e. a multidisperse system of clusters, aggregates, or islands, is given by naphthalene embedded in PMMA (below $20 \% \mathrm{wt} / \mathrm{wt}$ ). An example of the data is given in figs. $2-4$, for a $10 \%$ sample. We
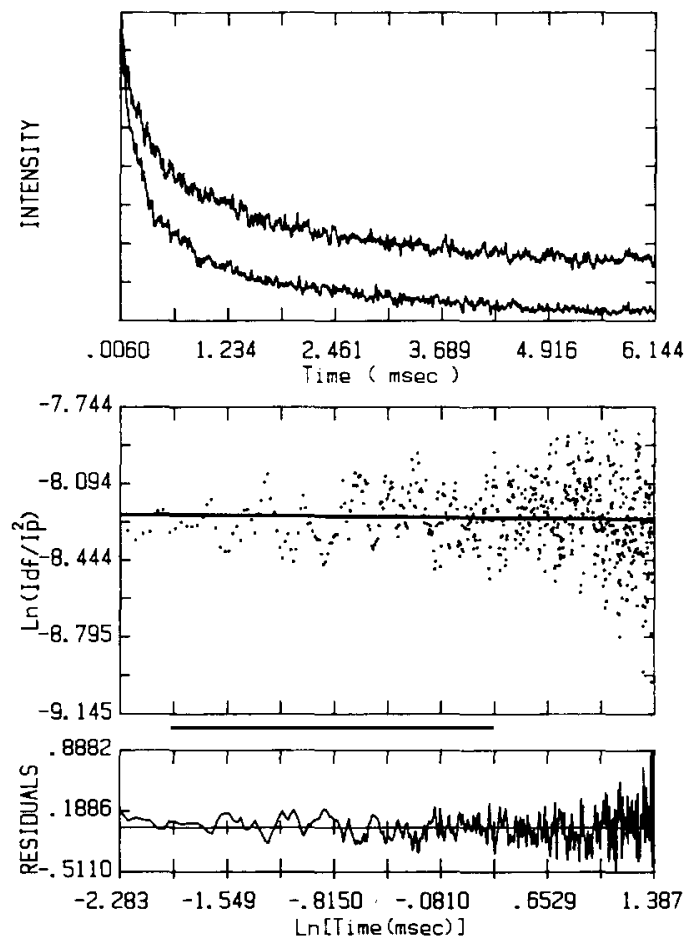

Fig. 2. $10 \%$ naphthalene/PMMA, $77 \mathrm{~K}$ pulsed excitation, $h=$ $0.0066 \pm 0.005$. Early time decay data (before $4 \mathrm{~ms}$ after the laser pulse). Homofusion fitting. 

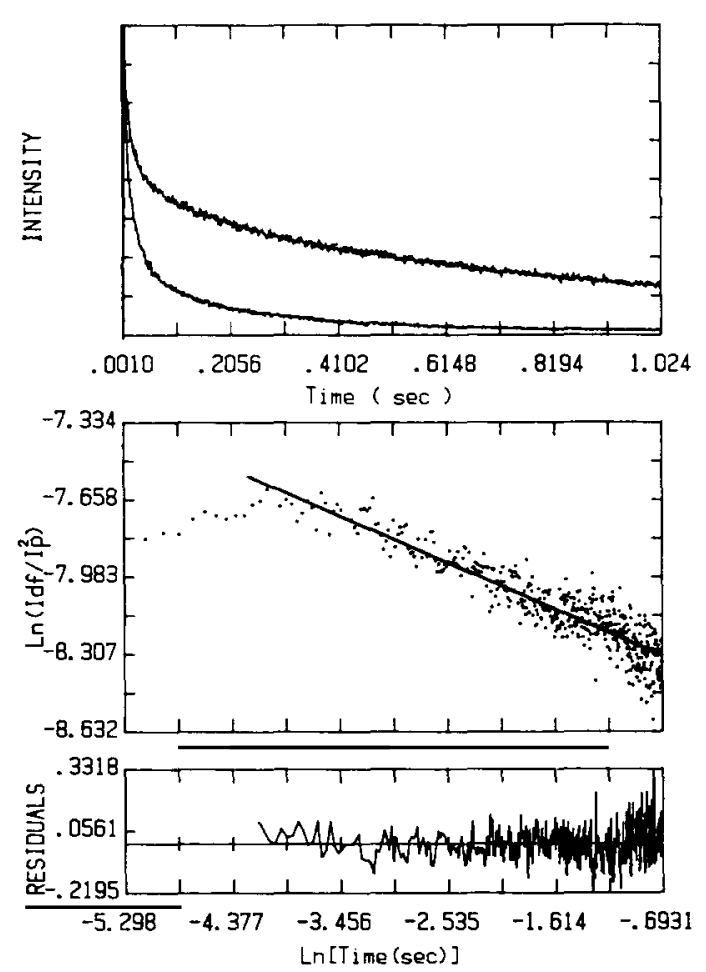

Fig. 3. $10 \%$ naphthalene/PMMA, $77 \mathrm{~K}$ pulsed excitation, $h=$ $0.21 \pm 0.08$. Later time decay data (about $10 \mathrm{~ms}$ to 1 or more seconds after laser pulse). Homofusion fitting.

relate the delayed fluorescence $F$ to the phosphorescence $P$ via the fractal-like kinetics relation of eq. (5). We note that for classical homofusion $h=0$. Table 1 gives $h$ for the early time decays (MS). Within the experimental uncertainty we find that $h=0$. Table 2 gives the same decays for longer times ( $\mathrm{ms}$ to $\mathrm{s}$ ). Here, only the $20 \%$ sample is consistent with $h=0$. The lower concentrations $(3 \%, 5 \%, 10 \%)$ have definite positive values in the range 0.2 to 0.4 (increasing with naphthalene dilution). Table 3 gives the results for the quasi-steadystate excitation (the decay is measured after a long sequence of pulses at $100 \mathrm{~Hz}$. The values of table 3 are in excellent agreement with those of table 2 . We note that the table 3 data are consistent with previous work [11], using real steady-state excitation (with a xenon lamp).

The early time data, giving $h=0$, are consistent with a random (Poissonian) distribution of excitons, created by random absorption of
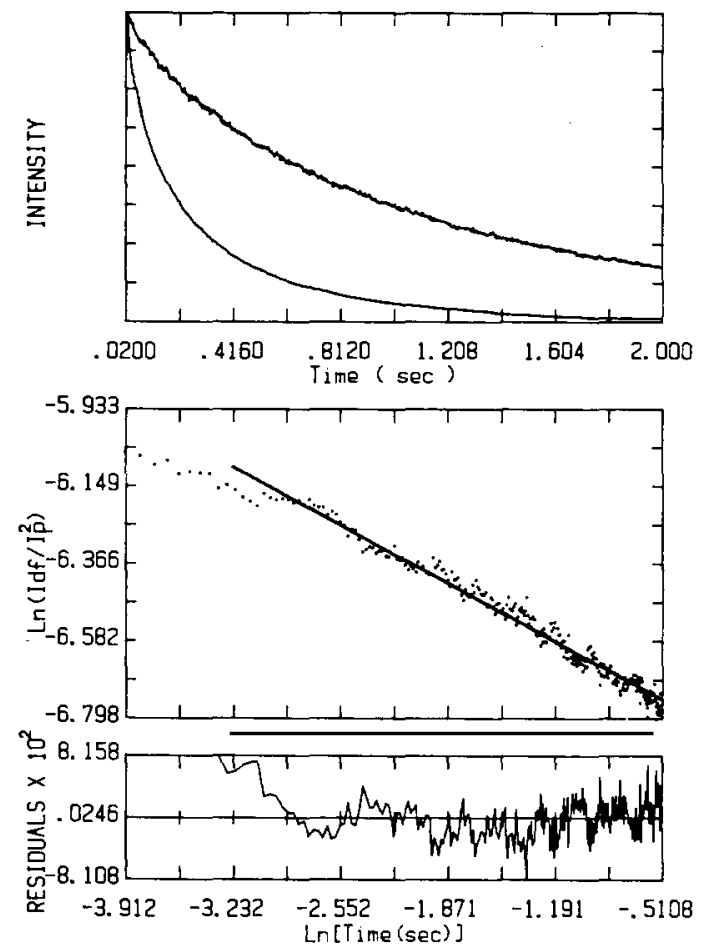

Fig. 4. $10 \%$ naphthalene/PMMA, $77 \mathrm{~K}, h=0.22 \pm 0.04$. Steadystate excitation by $100 \mathrm{~Hz}$ excimer laser. Data can also be considered as later time. Homofusion fitting.

photons. However, it has been shown $[2-4,8,11]$ for low-dimensional topologies (fractals, islands, clusters, and filaments) that a system of diffusing A particles that annihilate or fuse upon collision $(\mathrm{A}+\mathrm{A} \rightarrow 0$ or $\mathrm{A}+\mathrm{A} \rightarrow \mathrm{A})$ evolves towards a nonPoissonian stationary state. Specifically, the Hertzian distribution of nearest neighbor distances evolves towards a Wigner-like distribution (e.g.,

Table 1

Heterogeneities $^{\text {al) }}(h)$ vs. concentrations.

\begin{tabular}{ll}
\hline Concentrations $(w t / w t)$ & $h$ values \\
\hline $3 \%$ & $0.032 \pm 0.01$ \\
$5 \%$ & $0.018 \pm 0.01$ \\
$10 \%$ & $0.0066 \pm 0.005$ \\
$20 \%$ & $0.006 \pm 0.006$
\end{tabular}

a) These $h$ values were obtained by homofusion fittings, that is $-h$ is the slope of the plot of $\ln \left(I_{\mathrm{df}} / I_{\mathrm{r}}^{2}\right)$ vs. $\ln$ (Time) with early decay data (before $4 \mathrm{~ms}$ ). 
Table 2

Heterogeneities $^{\text {a) }}(h)$ vs. concentrations.

\begin{tabular}{ll}
\hline Concentrations (wt/wt) & $h$ values \\
\hline $3 \%$ & $0.36 \pm 0.08$ \\
$5 \%$ & $0.33 \pm 0.05$ \\
$10 \%$ & $0.21 \pm 0.08$ \\
$20 \%$ & $-0.04 \pm 0.04$ \\
\hline
\end{tabular}

a) These $h$ values were obtained by homofusion fittings, that is $-h$ is the slope of the plot of $\ln \left(I_{\mathrm{d} d} / I_{\mathrm{p}}^{2}\right)$ vs. $\ln$ (Time) with later decay data (10 $\mathrm{ms}$ up to $1 \mathrm{~s}$ ).

Table 3

Heterogeneities $^{a)}(h)$ vs. concentrations.

\begin{tabular}{ll}
\hline Concentrations (wt $/ w t)$ & $h$ values \\
\hline $3 \%$ & $0.35 \pm 0.04$ \\
$5 \%$ & $0.31 \pm 0.05$ \\
$10 \%$ & $0.22 \pm 0.04$ \\
$20 \%$ & $0.06 \pm 0.03$ \\
\hline
\end{tabular}

a) These $h$ values were obtained by homofusion fittings, that is $-h$ is the slope of the plot of $\ln \left(I_{\mathrm{d} f} / I_{\mathrm{p}}^{2}\right)$ vs. $\ln$ (Time) with steady state excitation by $100 \mathrm{~Hz}$ excimer laser.

eq. (2)), with a severe depletion of those neighbors close enough to react. Wigner-like distributions occur for both batch reactions (after a relatively short self-organization time) and for steady-state reactions. These new stationary distributions result in $h=\frac{1}{2}$ for filaments, $h=\frac{1}{3}$ for critical percolation clusters, and $h=1-d_{\mathrm{s} / 2}\left(d_{\mathrm{s}}<2\right)$ for fractals with spectral dimension $d_{\mathrm{s}}$. It is thus the topology of the medium that determines the extent of the depletion zone and the concomitant appearance of the heterogeneity exponent. This forms the connection between the medium's effective topology and the measured time exponent $h$. For instance, the $5 \%$ sample gives, roughly, $h=0.33$, which is the same as derived [3] for critical percolation clusters. On the other hand, the $20 \%$ sample gives $h=0$, which is consistent with three-dimensional Euclidean topologies, such as crystal lattices. The latter result is consistent with light scattering data [12] and Raman data [13], which attribute to this sample a structure dominated by micron sized crystalline domains.

\section{Acknowledgment}

This work was supported by NSF Grant DMR 8801120

\section{References}

[1] Y.S. Bai and M.D. Fayer, Chem. Phys. 128 (1988) 135.

[2] R. Kopelman, S.J. Parus and J. Prasad, Chem. Phys. 128 (1988) 209.

[3] R. Kopelman, Science 241 (1988) 1620.

[4] C.R. Doering and D. Ben-Avraham, Phys. Rev. Lett. 62 (1989) 2563.

[5] Z-Y. Shi and R. Kopelman, Mol. Cryst. Liq. Cryst. 183 (1990) 143

[6] C.S. Li and R. Kopelman, J. Phys. Chem. 94 (1990) 2135. Macromolecules 23 (1990) 2223.

[7] R. Kopelman, C. S. Li and Z-Y. Shi, J. Lumin. 45 (1990) 40.

[8] S.J. Parus and R. Kopelman, Mol. Cryst. Liq. Cryst. 175 (1989) 119

[9] G.W. Weiss, R. Kopelman and S. Havlin, Phys. Rev. A 39 (1989) 466.

[10] R. Schoonover and R. Kopelman, Mol. Cryst. Liq. Cryst. 183 (1990) 181.

[11] R. Kopelman, S.J. Parus and Z-Y. Shi, in: Dynamical Processes in Condensed Molecular Systems, eds. J. Klafter, J. Jortner and A. Blumen, (World Scientific, Singapore, 1989) p. 231

[12] E.I. Newhouse and R. Kopelman, Chem. Phys. Lett. 143 (1988) 106.

[13] Y.-E. Koo and R. Kopelman, J. Phys. Chem. 93 (1989) 1677. 